\title{
Graphene Membrane Encapsulation Platform for Multi-technique Spectromicroscopy of Wet Objects
}

Christopher Arble ${ }^{1}$, Hongxuan Guo ${ }^{2}$, Alessia Matruglio ${ }^{3,4}$, Lisa Vaccari ${ }^{4}$ and Andrei Kolmakov ${ }^{1}$

${ }^{1}$ Physical Measurement Laboratory, National Institute of Standards and Technology, Gaithersburg, Maryland, United States, ${ }^{2}$ SEU-FEI Nano-Pico Center, Key Laboratory of MEMS of Ministry of Education Southeast University, Nanjing, Jiangsu, China (People's Republic), ${ }^{3}$ Electrochemical Innovation Lab, Department of Chemical Engineering, University College London, London, England, United Kingdom, ${ }^{4}$ Elettra Sincrotrone, Trieste, Piemonte, Italy

* Corresponding author: Andrei.Kolmakov@ nist.gov

Many important biological, physical, and chemical processes take place in aqueous environments and thus warrants the investigation of these systems under realistic conditions. Accessing in-vivo conditions is the next generation of biological optical, electron and X-ray imaging that will provide valuable insight into structure-activity relationships of biomolecules within cellular operations [1]. However, the incompatibility of cells, which subsist in aqueous conditions, with the vacuum environments soft x-rays and electron spectrometers as well as low dose thresholds for beam damage, impedes the capability of imaging and spectroscopic studies under physiological conditions. Recent advances in applications of two dimensional (2D) materials, i.e. graphene, to separate the sample environment from UHV conditions in the analysis chamber have been made by employing 2D materials as molecularly impermeable, electron transparent membranes [2]. This approach to examine samples under operational conditions using various types of microscopies is an attractive method to investigate cellular functions.

Herein we report on development and tests of the multicompartment graphene encapsulated sample platform designed to be suitable for in-situ studies of biological samples with an array of analytical methods such as optical/fluorescence/Raman as well as scanning electron and X-ray microscopies. (Figure 1). Low-stress $\mathrm{Si}_{3} \mathrm{~N}_{4}$ coated silicon wafers were wet etched to form 100 x $100 \mu \mathrm{m}$ membranes which comprises the base of the platform. The platform lid was composed of an SU-8 film which was photolithography patterned with corresponding windows over which graphene layers were transferred. Samples can be deposited on the $\mathrm{Si}_{3} \mathrm{~N}_{4}$ window and become encapsulated by the graphene membrane once the lid is placed on the platform. The conformability of single and multilayer graphene sheets over the shape of a specimen allows it to be intimately confined and have its environment preserved while becoming suitable to be studied with conventional analytical techniques [3]. The encapsulation by graphene minimizes the volume of water retained platforming the probing volume, reducing the adsorption and scattering cross-section of the sample matrix while still providing cells a hydrating environment. Implementation of sample immobilization with a biocompatible patternable gel media allows for an extended cellular lifetime under measurement. Preliminary feasibility tests of graphene encapsulation of wet samples have been conducted with optical, SEM, TEM, Raman and FTIR microscopies. 

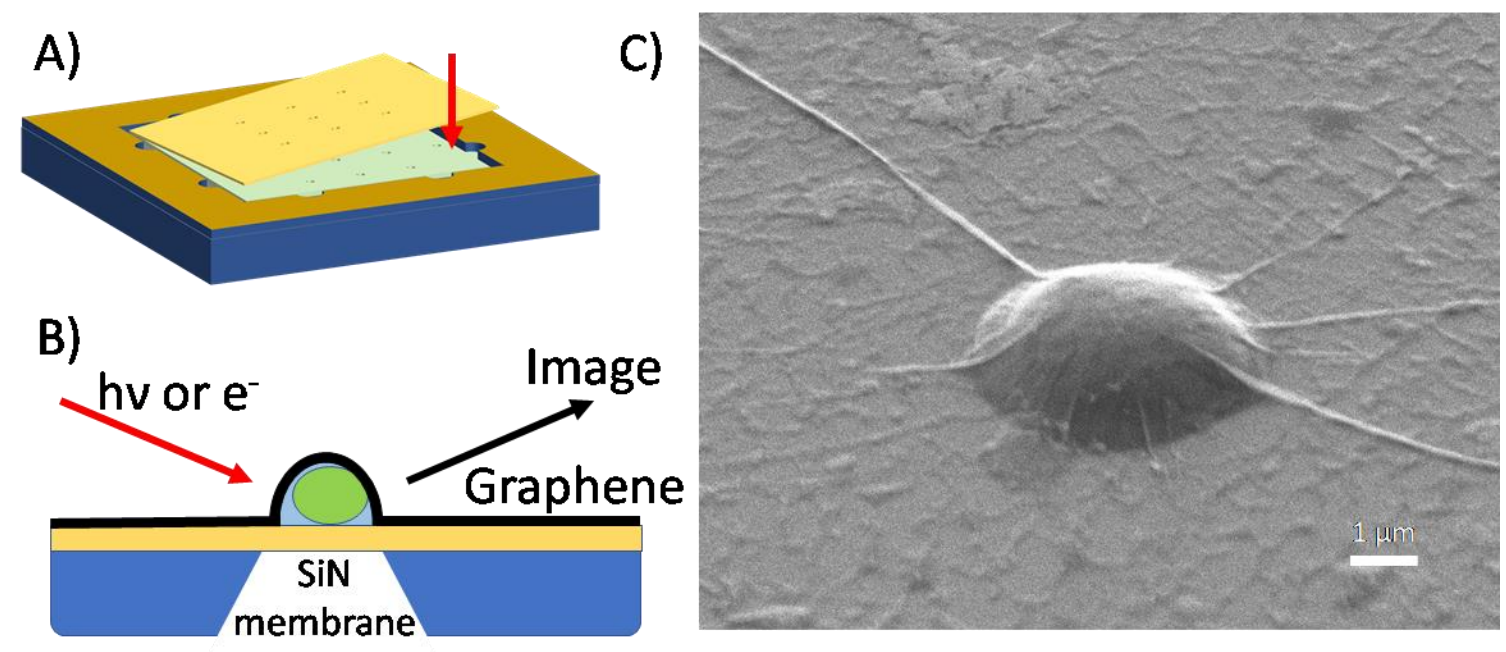

Figure 1. a) Liquid cell platform design b) Graphene encapsulation c) SEM image of encapsulated yeast cell

\section{References}

1. S. Sahl et al, Nature Reviews Molecular Cell Biology 18 (2017) p.685-701

2. A. Kolmakov et al, Nature Nanotechnology 6 (2011) p. 651.

3. A. Yulaev et al, Advanced Materials Interfaces, 4, (2016) p.1600734 